\title{
Post Purchase Search Engine Marketing
}

\author{
Qianyun Zhang \\ New York University \\ New York, New York, US \\ qianyunzhang@nyu.edu
}

\author{
Shawndra Hill \\ Microsoft Research \\ New York, New York, US \\ shawndra@microsoft.com
}

\author{
David Rothschild \\ Microsoft Research \\ New York, New York, US \\ davidmr@microsoft.co
}

\begin{abstract}
Although consumer behavior in response to search engine marketing has been studied extensively, few efforts have been made to understand how consumers search and respond to ads post purchase. Advertising to existing customers the same way as to prospective customers inevitably leads to wasteful and inefficient marketing. Employing a unique dataset that combines both search query and purchase data, we examine consumers' searching behavior and response to search engine marketing after purchase. We study large advertising campaigns for two popular technology products. We find that over half of the branded keyword searches come from consumers who already purchased the products, and that advertising response varies based on whether searchers are pre- or postpurchase. In general, post-purchase searchers are less likely to click on focal brand ads (i.e., they are less responsive to ads for products they already own). However, post-purchase searchers are still responsive to advertising and much more likely to click on ads for complementary products (i.e., they are more responsive to ads for relevant products other than the focal product).
\end{abstract}

\section{KEYWORDS}

online advertising; search engine; post-purchase; purchase funnel

\section{ACM Reference Format:}

Qianyun Zhang, Shawndra Hill, and David Rothschild. 2018. Post Purchase Search Engine Marketing. In WWW'18 Companion: The 2018 Web Conference Companion, April 23-27, 2018, Lyon, France. ACM, New York, NY, USA, 8 pages. https://doi.org/10.1145/3184558.3186583

\section{INTRODUCTION}

In the last 20 years, search engine marketing has grown epidemically. Since the advent of sponsored search advertising, sponsored ads have accounted for a majority of online advertising expenditure. It is estimated that over half of Google's revenue in 2016 came from sponsored ads [15]. It is generally accepted that search engine marketing is more targeted than other means of digital advertising because consumers have just shown interest in a particular product (by searching for it), thus limiting "wasted expenditure" on potential consumers who have not explicitly shown interest [2], [7].

Still, some waste is expected as advertisers try to attract consumers very early in the conversion funnel, which describes the journey as consumers navigate online sites, from the first step of awareness of a product to a final purchase decision [18]. The idea is

This paper is published under the Creative Commons Attribution 4.0 International (CC BY 4.0) license. Authors reserve their rights to disseminate the work on their personal and corporate Web sites with the appropriate attribution.

WWW' 18 Companion, April 23-27, 2018, Lyon, France

() 2018 IW3C2 (International World Wide Web Conference Committee), published under Creative Commons CC BY 4.0 License.

ACM ISBN 978-1-4503-5640-4/18/04.

https://doi.org/10.1145/3184558.3186583 that at the top of the funnel, there are many options; at the bottom of the funnel, there are fewer, more specific options narrowed down for potential purchase by the consumer. Advertisers often bid on a variety of keywords, including branded keywords (those related to the focal brand) and generic keywords (those related to the general product category) [26]. It is widely accepted that consumers in early stages of the conversion funnel will search for generic keywords while consumers closer to making a purchase will search for a specific focal brand [17]. Researchers have already suggested that consumers make different queries and respond differently to ads depending on their purchase funnel stage [17].

For example, consumers who search for generic keywords such as "computers" are likely to be at the consideration or awareness stage while consumers who search for "Lenovo computers" are assumed to be at the consideration stage and closer to making a purchase. Consequently, advertisers of the focal brand (in this example, Lenovo) would typically pay more attention to consumers searching for "Lenovo computers" and tend to bid heavily on focal branded keywords. Thus, on average, branded keywords are priced higher than generic ones [29], [3].

The two examples of sponsored search ads in Figure 1 illustrate how strategies that advertise to post-purchase searchers the same way as to pre-purchase searchers are flawed. The first example is one author searching for "Lenovo T460" on Bing.com from a Lenovo T460 computer. Bing presents an ad attempting to sell another T460 computer. The second example is an author searching for Windows 10 on Google from a computer with Windows 10 installed. In both examples, the advertisers treat the searcher as of they are very close to making a purchase when in reality they are not. These examples indicate that this underlying assumption may lead advertisers to waste their budgets by showing customers products they already own. In this study, we argue that by ignoring post-purchase search, online advertisers do not achieve full efficiency with a large portion of their branded searches. A better understanding of consumers' post-purchase information-seeking behavior can help companies strategically plan and provide consumer services, which will improve consumer satisfaction, and likely revenue, in the long run.

In fairness to prior work, which has focused exclusively on prepurchase search, it is very difficult to acquire both search and purchase data in the same place. However, solutions to exclude lists of users now exist (e.g., a list of existing customers). Beginning in 2017, Bing, Google, and Facebook launched new functions that advertisers can use to distinguish consumers who have converted from those who have not. While the data labels on whether someone is an existing customer are still noisy, advertising differently to customers is now possible. Still, these companies give little advice on what to do with the data: Should advertisers continue to market the same way? Should they not market at all? Or should they advertise complementary products when possible? 


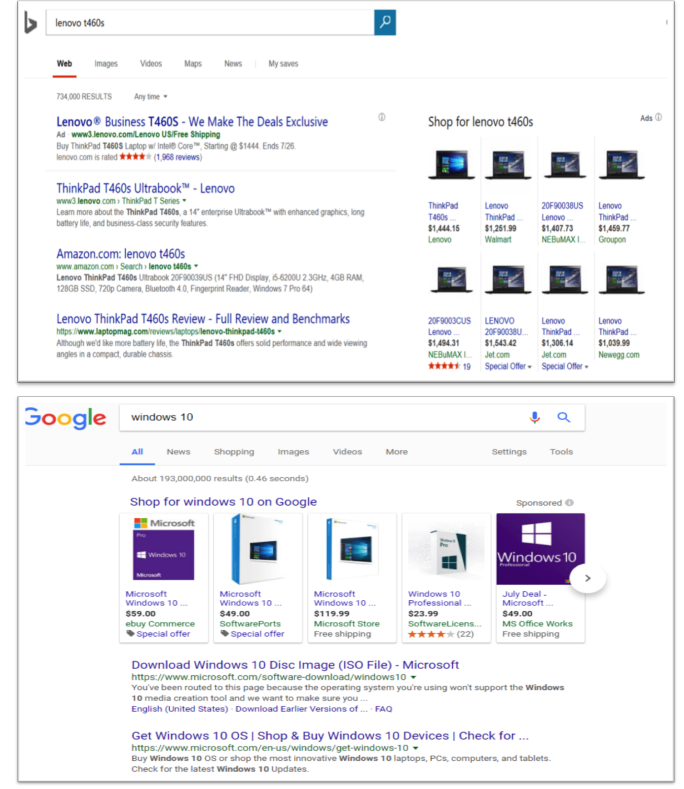

Figure 1: Examples of Sponsored Ads

Our study aims to answer these questions by examining consumers' post-purchase searches on the Bing search engine. In general, post-purchase searchers are less likely to click on focal brand ads-they are less responsive to ads for products they already own. However, post-purchase searchers are much more likely to click on ads for complementary products-they are more responsive to ads for relevant products other than the focal product. From the perspective of the search engine, they are also more likely to click on the search results page when an ad is shown. We contribute to the literature by empirically investigating consumers' needs after purchase and discussing how to strategically advertise to post-purchase searchers. Our findings also bear important practical implications to sponsored search advertisers and search engine designers.

\section{RELATED WORK}

Our work is positioned at the intersection of two literatures in which there is extensive work: information seeking and sponsored search advertising. Due to space constraints, we hyperfocus this literature review on work related to identifying search intent and ad response in relation to the conversion funnel. While there has been significant work exploring how people search at various points in the conversion funnel pre purchase, we have found that prior research has completely ignored the fact that consumers both seek information and are exposed to sponsored search ads post purchase.

\subsection{Search Intent}

Early work on online consumers in the purchase context found that people have different goals when surfing the web. Over time these goals have been translated to search intent. An early study by Broder [4] found that there are mainly three types of search intent, which correspond to three types of search queries: informational, navigational, and transaction-related queries. Moe [24] examined browsing data to identify a typology of consumers' store visits. Four types of store-visit goals were identified: buying, browsing, searching, and knowledge building. The author found that browsing alone accounts for around $60 \%$ of visits, searching for around $30 \%$, and buying for around 7\%. The most relevant aspect of this work to our study is that the authors found heterogeneity in purchase intent depending on the context. Consumers with different goals demonstrate various likelihoods to purchase and respond differently to various marketing messages.

This early study was followed by a growing body of literature classifying consumers' search intents. Jansen, Booth and Spink [16] and Kathuria, Jansen, Hafernik and Spink [19] found that the majority of search queries are informational, although there are substantial navigational queries.

Another growing body of literature is focused on combining queries with consumer click-stream behavior to automatically identify consumers' search intents [22], [16], [10],[19],[14], [25]. Various methods such as k-means clustering and supervised vector machine have been employed for automatic identification and prediction of intent. Jansen and Schuster [17] further integrated traditional conversion funnel theory into the classification of search queries and found distinct differences in involvement between stages; consumers show various levels of interest in a product depending on their funnel stage identified by a predictive model. Recent work has found that consumers also have multiple search intents, even within a single search query. Gonzales-Caro and Baeza-Yates [8] and Kathuria et al. [19] found that queries are likely to be multifaceted.

\subsection{Sponsored Search Advertising Response}

With sponsored search advertising gaining in popularity among marketers, early academic work focused on quantifying the benefit of using sponsored search advertising in terms of ROI, compared to other traditional marketing channels. A comprehensive study by Ghose and Yang [7] empirically analyzed the relationship between search engine query metrics such as page position, click-through rates, and conversion profits. Chan, $\mathrm{Wu}$, and Xie [5] examined the overall lifetime value of consumers acquired from online and offline conversion channels and found that consumers acquired from sponsored search ads have higher transaction rates and longterm value, accounting for spillover effects. However, it has been argued that sponsored search ads are likely to be over-credited under the last-click attribution model [23].

As sponsored search ads gain more recognition among marketers, researchers are shifting their focus and analyzing one or two aspects of either the platform (what is being shown to users), advertisers' bid strategies, or different outcomes to identify recommended best practices for advertisers. Sponsored search ads often interact with other aspects of the search results page. Yang and Ghose [31] investigated the dependence between sponsored search ads and organic search results, and their findings suggest that appearance in organic search results can enhance the effectiveness of sponsored search advertising. Other studies have focused on branded keywords. Rutz, Trusov, and Bucklin [27] found that consumers who search branded or vague keywords are more likely to revisit a store. Using a large-scale field experiment, Blake, Nosko, and Tadelis [3] found that branded keyword ads have very small 
to no short-term returns. When a company stops advertising, consumers who search for its branded keywords use organic listings to find the information they need. Contrary to common belief, the return for sponsored search advertising is, on average, negative [3]. Simonov, Nosko, and Rao [28] further investigated the primary value of branded keyword ads and found that when a company stops advertising for its focal brand keyword, some users are likely to be stolen by its competitors. Thus, brand keyword ads should be used primarily to defend existing market shares.

Another growing stream of literature is focused on predicting searchers' satisfaction with search engine results [21], [20], [13], [11], [6], [12], [30]. Click-through rates and dwell time on search engine results pages have been determined as two important indicators for prediction, and our analysis relies on these two metrics.

\section{DATA}

We use search data generated from users of the Bing search engine tied to purchase data.

\subsection{Search Data}

We filter the search queries by branded keywords (i.e., queries that include the terms "Windows 10" or "Xbox One"). We employ not only filtered search queries but also search engine results (ad links and organic links), clicks on the results page, and dwell time on the page. The main reason we focus on branded queries is because they include well-balanced searches from both pre- and post-purchase users.

We focus on two products for this study: Windows 10 and Xbox One. The data we collect to describe the differences in overall search intent includes Bing searches for Windows 10 during its launch campaign from July to October 2015 and searches for Xbox during November and December 2016. The data we collect for analyzing sponsored search ad response is from April to June 2017. We use more recent data for the advertising analysis because we rely on random flight data ${ }^{1}$ that are stored for only the most recent months.

Since the focal product ad link is usually the most relevant to a focal brand search (e.g., when someone searches for Xbox One S, the most relevant link is likely the ad link to the Microsoft store, which sells Xbox), it is hard to determine with the current Bing algorithm, which shows the same thing to everyone, what would happen if a non-focal ad is shown or if no ads are shown (in other wordsm we have no counterfactuals). However, there is a small random flight on Bing that randomly selects which and how many ads are shown for a very small percentage of search traffic. The random flight data enable us to ask questions about how users will respond when they are shown non-focal brand products. We can also use these data to restrict results to cases when only one ad is shown.

Figure 2 is a bar chart of the ads shown for the specific query "Windows 10" for both regular and random flights. It shows that the large majority of users see the same one ad with the regular flights - an ad for Windows 10 (plot for Xbox omitted due to space constraints). However, for the random flights, the ads shown to

\footnotetext{
${ }^{1}$ Flights are identifiers for groups of queries that are being shown results based on the same algorithm. The random flight uses a randomized algorithm to select the number of ads and rank at random a set of relevant ads and is thus a randomized experiment.
}

searchers are distributed over many more ad links. This variation due to experimentation allows us to answer questions regarding which types of ads are more impactful for post-purchase searchers. These random flights allow us to set up our data as if it came from an experiment. It is important to note that Bing ads for Windows 10 and Xbox One products do not differentiate searchers based on the device used or other sales-related information.

Figure 2: Advertisements on Windows 10 for Regular and Random Flights

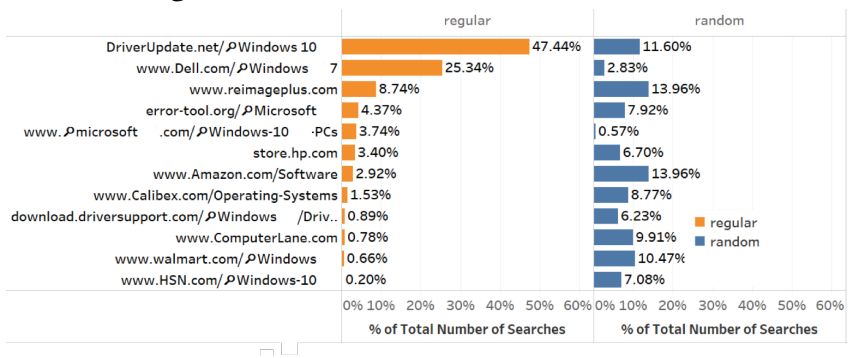

\subsection{Purchase Data}

For Windows 10, to identify whether a search was made pre- or post-purchase, we label searches from Windows 10 devices as postpurchase searches. For Xbox, we rely on anonymous Microsoft product sales data. If a consumer searched before activating the product, we categorize the search as pre-purchase. If a consumer searched after activating the product, we categorize the search as post-purchase.

We find that over half of the searches with a keyword containing the focal brand name are from users who have already purchased (52\% in the case of the first nine weeks of the Windows 10 launch and $68 \%$ in the case of the Xbox One holiday season from November to December 2016).

\section{RESULTS}

In this section, we first examine the differences between pre- and post-purchase searches in general for both the Windows 10 product launch and Xbox during the holiday sales season of 2016. We investigate how pre- and post-purchase searchers differ in their search intents and then look at how they differ in what they click on and click-through rates. We then focus on answering our research question, namely "What should an advertiser advertise to existing customers?" We rely on response to sponsored search advertising for just the focal brands (the exact search "Windows 10" and the exact search "Xbox One"), utilizing the random flight data, which includes ad impressions for both the focal brand ads and relevant but non-focal brand ads.

\subsection{Search Intent}

4.1.1 Windows 10. At the beginning of the launch of the Windows 10 campaign, most searches for Windows 10 came from users who had not upgraded; however, as the campaign progressed, the proportion of searches from Windows 10 users quickly rose, and a 
majority of searches for Windows 10 came from existing customers. In addition, the number of overall searches quickly declined one month after the product launch, showing that interest in a product quickly declines after people have been made aware of it via a product launch. For Windows 10, we analyze searches containing "Windows 10" during the product launch campaign from July to October 2015. Figure 3 plots the linear standardized number of searches containing "Windows 10" across the first few months after the product launch. The orange represents post-purchase searches. While pre-purchase searches are mainly focused on "Windows 10 " and "upgrade", post-purchase searches are focused more on troubleshooting Windows 10.

Figure 3: Searches for Windows 10 during the First Nine Weeks of Product Launch

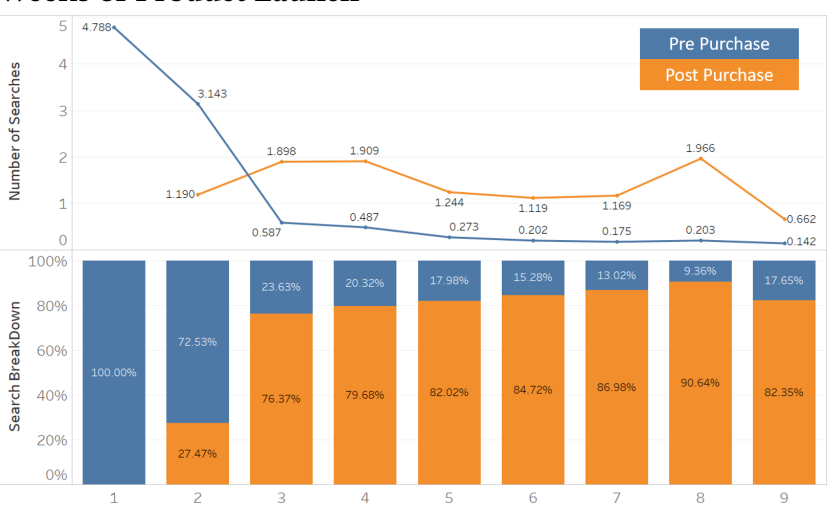

Within top search queries (i.e., conditional on searching for the exact same thing), post-purchase searchers are less likely to click on ads compared to pre-purchase searchers. One explanation for this is that most ads are tailored to pre-purchase searchers so it is likely that post-purchase searchers do not find the ads useful or helpful. Figure 4 plots ad click-through rates for each search query for pre- and post-searches. To make this metric comparable between searches, we focus on cases where searchers see only two ads. The queries here are all top search queries based on number of searches. The orange represents post-purchase searches. Similar analyses were repeated for other products, and the results are presented in the following subsections.

4.1.2 Xbox. Similar to Windows 10, people search for different topics about Xbox, pre and post purchase. This section discusses findings for searches that contain "Xbox" from November to December 2016. Also similar to Windows 10, post-purchase searchers are more likely to search for troubleshooting information. Current Xbox customers' click-through rates are consistently lower, whether from existing customer-dominated queries (like those searching for help) or non-customer-dominated queries, than pre-purchase searchers. This further confirms our observation from Figure 4 that post-purchase searchers are less likely to click on ads compared to pre-purchase searchers. Similar to Figure 4, Figure 5 plots ad click-through rates for each search query, conditional on searchers seeing two ads. For Xbox, we also examine the dynamics of the search. For each consumer who purchased an Xbox during the last
Figure 4: Top Search Queries for Windows 10 and Clickthrough Rates

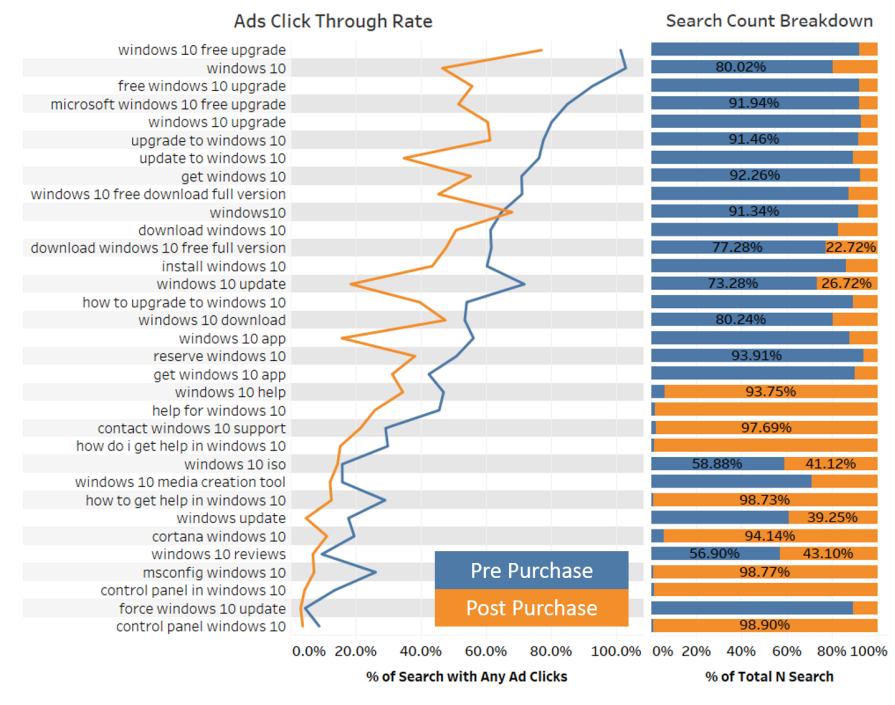

Table 1: Percentage of Consumers Who Searched Before and After Purchase

\begin{tabular}{ccc}
\hline $\begin{array}{c}\text { Pre-purchase Search } \\
\text { (30 days before) }\end{array}$ & $\begin{array}{c}\text { Post-purchase Search } \\
\text { (30 days after) }\end{array}$ & $\begin{array}{c}\text { Percentage } \\
\text { of Users }\end{array}$ \\
\hline No & Yes & $62 \%$ \\
Yes & No & $26 \%$ \\
Yes & Yes & $12 \%$ \\
\hline
\end{tabular}

week of November or first week of December 2016, we analyze how they searched before and after the purchase. From Figure 6, we plot the number of consumers who searched and the relative date to purchase. As can be seen, consumers made the most searches one day before purchase and then continued to search after purchase. A long tail in search can be observed after purchase. To further understand pre- and post-purchase search behavior, we examine how many consumers searched only before or only after purchase. The results are reported in Table 1. Interestingly, in our data, only $12 \%$ of consumers searched both before and after purchase; the majority of consumers searched only after purchase.

\subsection{Sponsored Search Ad Response}

4.2.1 Overall. In this section, we analyze how searchers respond differently to ads for all selected queries by examining two metrics: click-through rate and dwell time on the search engine results page. These metrics have been widely used in the information retrieval literature to measure how satisfied searchers are with search engine results. In our analysis, we constrain searches to those in which searchers see exactly two ads at the top of the search engine results page. Our analysis compares how pre- and post-purchase searches differ in these two metrics, conditional on searching for the same query and seeing the same ads during the same time period. We 
Figure 5: Top Search Queries for Xbox and Ad Click-through Rates

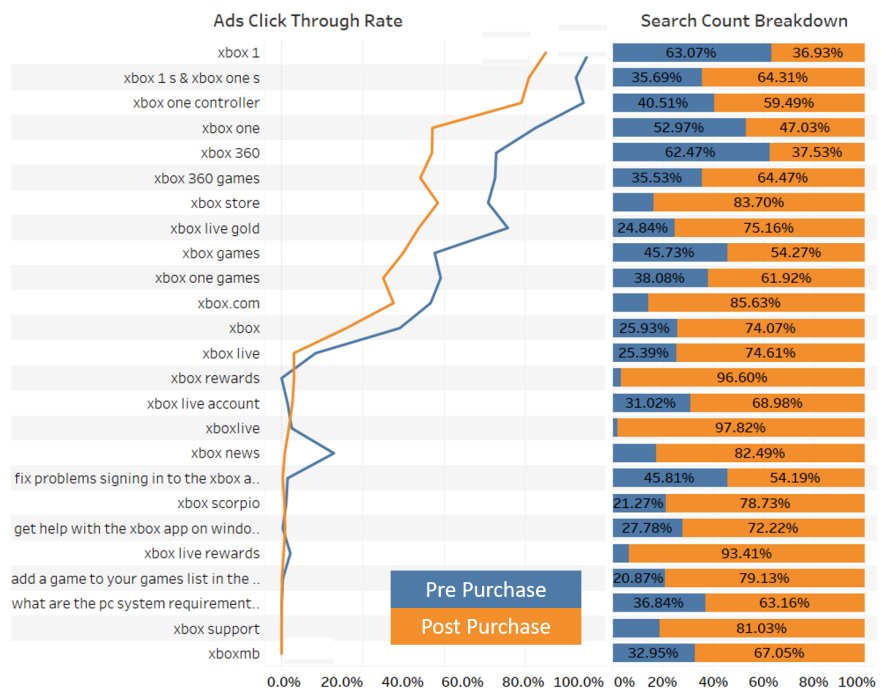

Figure 6: Searches Pre- and Post-purchase

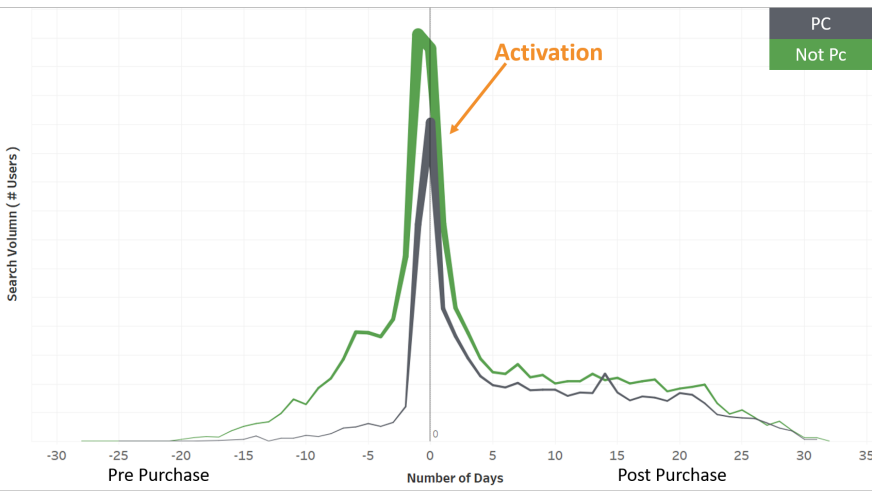

focus our analysis on queries with as least 10 pre-purchase searches and 10 post-purchase searches. The t-test results are displayed in Table 2.

Results for both Windows 10 and Xbox suggest that post-purchase searchers spend less time examining the ads on search engine results pages and are less likely to click on ads compared to pre-purchase searchers. Accordingly, the difference in click-through rates and dwell time on the search engine results page suggests that current product users quickly move on to other information on the results page when they find the ads do not meet their needs.

When searchers do not find ads useful, they are likely to use organic results to meet their needs. We compare the click-through rates and dwell times for organic results between pre- and postpurchase searches, conditional on searching for the same query and seeing the same organic and ad results in the same time period. Table reports the statistics and test results. On average, current product users spend more time looking at and are more likely to click on organic search results compared to non-users. Thus, postpurchase searchers are more likely to be satisfied with organic results.

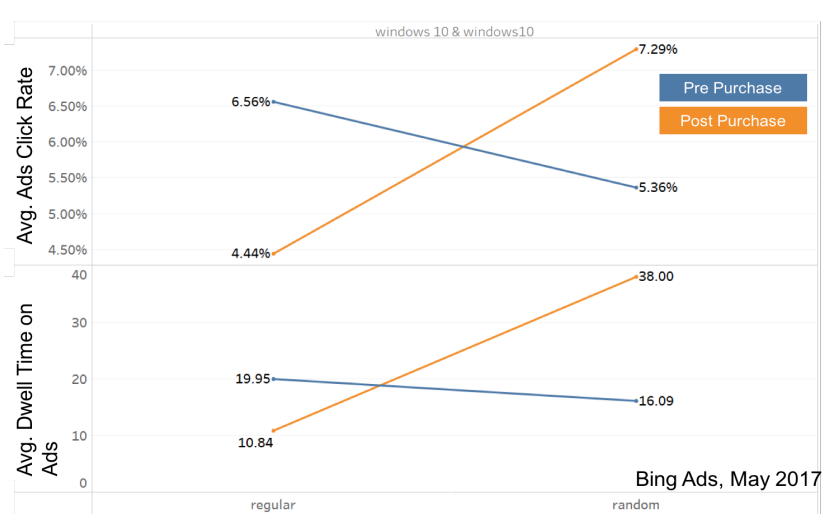

Figure 7: Ad Click-through Rates and Dwell Times for Regular versus Random Flights for Pre- and Post-purchase Users

4.2.2 Specific Ad Response: "Windows 10" and "Xbox One". The findings from previous sections indicate that post-purchase searches are made to fulfill needs distinct from those that motivate prepurchase searches. In this section, relying on the variation in ads shown from the random flights, we investigate which type of ad does a better job of satisfying post-purchase searchers' needs. We focus on two search queries: "Windows 10" and "Xbox One" using data from Bing ads in 2017. For Windows 10, 30 days of data was collected during May 2017. For Xbox, 60 days of data was collected from April to June 2017. During the period we observe, ads for Windows 10 and Xbox varied due to competition and advertisers' strategic bidding behavior. This provides the variation in advertisement that we need to identify which types of ads better satisfy post-purchase search needs. It is possible that this variation is subject to certain advertising bias but according to current Bing ad bidding rules, advertisers (i.e., Dell or Microsoft) cannot distinguish between pre- and post-purchase searchers. Thus, this variation equally influences both pre- and post-purchase searches. To control for unobserved bias, we add controls for demographics and geographic location, which are the two most frequently used factors by ads to segment searchers. Figure 7 shows model-free evidence that these groups of users respond differently to different types of ads. We see that existing users are less likely to click on ads in the regular flights but more likely to click on ads in the random flights, which include many more complementary products. To demonstrate this difference more formally, we label the ads and also run a regression to quantify the size of the effect of showing complementary ads versus focal brand ads to pre- versus post-purchase searchers.

Our analysis includes two types of sponsored search ads: ads related to the focal brand and non focal brand ads. We use several rules to determine whether an advertisement is a focal brand ad. For Windows 10, we categorize advertisements that meet any of the following rules as non focal brand ads: mentions driver update, reviews, system repair, fixing errors, or anti-virus in the advertisement message. 


\begin{tabular}{rlrrrrr}
\hline Product & Metric & Post & Pre & Post-Pre & Sta & P-value \\
\hline Windows 10 & Click-through Rate & 0.06 & 0.09 & -0.03 & -13.83 & 0.00 \\
Windows 10 & Dwell Time & 9.57 & 13.27 & -3.70 & -4.03 & 0.00 \\
Xbox & Click-through Rate & 0.16 & 0.20 & -0.04 & -6.06 & 0.00 \\
Xbox & Dwell Time & 24.75 & 30.37 & -5.62 & -3.36 & 0.00 \\
\hline
\end{tabular}

Table 2: Differences in Advertising Metrics for Pre- and Post-purchase Users

\begin{tabular}{rlrrrrr}
\hline Product & Metric & Post & Pre & Post-Pre & Sta & P-value \\
\hline Windows 10 & Click-through Rate & 0.48 & 0.48 & 0.00 & 0.22 & 0.83 \\
Windows 10 & Dwell Time & 88.22 & 82.39 & 5.83 & 2.15 & 0.03 \\
Xbox & Click-through Rate & 0.40 & 0.37 & 0.03 & 3.80 & 0.00 \\
Xbox & Dwell Time & 76.29 & 65.91 & 10.38 & 3.44 & 0.00 \\
\hline
\end{tabular}

Table 3: Differences in Organic Click Metrics for Pre- and Post-purchase Users

For Xbox, we categorize advertisements that meet any of the following rules as non focal brand ads: mentions $x$ box live, $x b o x$, gold, video game, controller, hard drive, headset, or any other obvious accessories in the advertisement message.

We first investigate whether ads should be shown in the results for post-purchase searches by comparing cases where only one advertisement is shown to cases where users do not see an advertisement. We model whether a user clicks on anything on the results page, including both organic results and sponsored search ads, as a binary choice as a function of search type (i.e., pre- or post-purchase) and whether an advertisement is shown (at all). We then investigate, conditional on showing an ad, what kind of ads would work best for these searches. We focus on cases where only one ad is shown on the results page. To answer these two questions, we use the following two models for estimation:

$$
\begin{aligned}
& \text { Click }_{i}=\text { UserType }_{i}+\text { ShowAds }_{i}+\text { ShowAds }_{i} * \text { UserType }_{i} \\
& \text { Click }_{i}=\text { UserType }_{i}+\text { AdsType }_{i}+\text { AdsType }_{i} * \text { UserType }_{i}
\end{aligned}
$$

\begin{tabular}{|c|c|c|c|}
\hline $\begin{array}{r}\text { Search } \\
\text { Type } \\
\end{array}$ & $\begin{array}{l}\text { Number of } \\
\text { arches Per User }\end{array}$ & $\begin{array}{l}\text { Percentage of Searches } \\
\text { Shown Sales Ads }\end{array}$ & $\begin{array}{l}\text { s Ad Click- } \\
\text { through Rate }\end{array}$ \\
\hline $\operatorname{Pr}$ & 1.27 & 1.24 & 4.77 \\
\hline Post & 1.27 & 1.85 & 2.77 \\
\hline
\end{tabular}

Table 4: Windows 10 Descriptives

\begin{tabular}{|c|c|c|c|}
\hline $\begin{array}{r}\text { Sear } \\
\text { Typ } \\
\end{array}$ & $\begin{array}{l}\text { Number of } \\
\text { arches Per User }\end{array}$ & $\begin{array}{l}\text { Percentage of Searches } \\
\mathrm{r} \quad \text { Shown Sales Ads }\end{array}$ & $\begin{array}{l}\text { s Ads Click- } \\
\text { through Rate }\end{array}$ \\
\hline $\operatorname{Pr}$ & 1.4 & 93.78 & 1.67 \\
\hline Post & 4.33 & 94.31 & 0.11 \\
\hline
\end{tabular}

Table 5: Xbox Descriptives

Table 4 presents the results of descriptive analysis for the data on searches for Windows 10, and Table 6 presents the estimation results for Windows 10 . As we can see from Table 4, only a small portion of searches are shown focal brand ads for Windows 10 .
This is understandable given that Windows 10 was released about two years ago. On average, post-purchase searches are more likely to be shown focal brand ads. This validates that advertisers cannot distinguish between pre-purchase searches and post-purchase searches. As can be seen, post-purchase searches also have lower click-through rates for advertisements.

The first column of Table 6 presents the estimation results regarding whether post-purchase searches should be shown ads. The negative coefficients for post-purchase search indicate that, on average, post-purchase searchers are less likely to click on anything on the results page, including both organic results and ads. However, the positive coefficient for the interaction between user type (post purchase or not) and advertisement type (focal brand or not) indicates that showing ads to post-purchase searches can improve the overall click-through rate on results pages. The second and third columns answer the question of what types of ads should be shown to post-purchase searchers. The negative coefficient for post-purchase confirms our finding in previous sections: post-purchase searchers have lower advertisement click-through rates. The positive, though not significant, coefficient for "NonFocalBrand" suggests that searchers are more likely to click on ads when they are for a non focal brand (or complementary product). The positive coefficient for the interaction term indicates that postpurchase searchers are more likely to click when the ad is not a focal brand ad. The difference between Model 2 and Model 3 is that in Model 3, we add controls for major domains such as Amazon, Best Buy, and Microsoft. These domain effects absorb some effects from the interaction term. The third column of Table 6 also adds demographics such as gender and geographic location. In Model 3, while the coefficient for interaction is still positive, it is not significant. Tables 5 and 7 present similar descriptive analyses and estimation results for data on searches for Xbox. Xbox estimation results are similar in direction and, in addition, all results are significant.

Our estimation results suggest that from a search engine perspective, post-purchase searchers should be shown ads. From an advertiser's perspective, non focal brand ads best serve post-purchase searchers' needs based on the click-through rate. This result corresponds to the widely recognized heterogeneity in advertisement effects [1]. For experienced consumers (those who know the brand 
Table 6: Windows 10 Estimation

\begin{tabular}{|c|c|c|c|}
\hline & \multicolumn{3}{|c|}{ Dependent variable: } \\
\hline & \multirow{2}{*}{$\begin{array}{c}\text { ClickAnything } \\
\text { (1) }\end{array}$} & \multicolumn{2}{|c|}{ ClickAds } \\
\hline & & $(2)$ & (3) \\
\hline Constant & $\begin{array}{c}0.367^{* * *} \\
(0.024)\end{array}$ & $\begin{array}{c}-3.420^{* * *} \\
(0.384)\end{array}$ & $\begin{array}{l}-1.550 \\
(1.099)\end{array}$ \\
\hline Post & $\begin{array}{c}-1.143^{* * *} \\
(0.031)\end{array}$ & $\begin{array}{c}-2.499^{* *} \\
(1.072)\end{array}$ & $\begin{array}{c}-2.302^{* *} \\
(1.077)\end{array}$ \\
\hline ShowAds & $\begin{array}{c}0.180^{* * *} \\
(0.030)\end{array}$ & & \\
\hline Post ${ }^{\star}$ ShowAds & $\begin{array}{l}0.088^{* *} \\
(0.041)\end{array}$ & & \\
\hline NonFocalBrand & & $\begin{array}{c}0.431 \\
(0.386)\end{array}$ & $\begin{array}{l}-0.002 \\
(0.449)\end{array}$ \\
\hline Post ${ }^{\star}$ NonFocalBrand & & $\begin{array}{l}1.947^{*} \\
(1.074) \\
\end{array}$ & $\begin{array}{c}1.724 \\
(1.079) \\
\end{array}$ \\
\hline Domain Effects & No & No & Yes \\
\hline Log Likelihood & $-28,202.120$ & $-5,954.415$ & $-5,806.082$ \\
\hline
\end{tabular}

Table 7: Xbox Estimation

\begin{tabular}{|c|c|c|c|}
\hline & \multicolumn{3}{|c|}{ Dependent variable: } \\
\hline & \multirow{2}{*}{$\begin{array}{c}\text { ClickAnything } \\
\text { (1) }\end{array}$} & \multicolumn{2}{|c|}{ ClickAds } \\
\hline & & $(2)$ & (3) \\
\hline Constant & $\begin{array}{c}-0.755^{* * *} \\
(0.020)\end{array}$ & $\begin{array}{c}-4.093^{* * *} \\
(0.084)\end{array}$ & $\begin{array}{c}-7.391^{* * *} \\
(1.493)\end{array}$ \\
\hline Post & $\begin{array}{c}-0.801^{* * *} \\
(0.032)\end{array}$ & $\begin{array}{c}-3.188^{* * *} \\
(0.507)\end{array}$ & $\begin{array}{c}-3.673^{* * *} \\
(0.580)\end{array}$ \\
\hline ShowAds & $\begin{array}{c}0.498^{* * *} \\
(0.032)\end{array}$ & & \\
\hline Post ${ }^{*}$ ShowAds & $\begin{array}{c}0.092 \\
(0.063)\end{array}$ & & \\
\hline NonFocalBrand & & $\begin{array}{c}0.239 \\
(0.304)\end{array}$ & $\begin{array}{c}-0.760^{* *} \\
(0.381)\end{array}$ \\
\hline Post ${ }^{\star}$ NonFocalBrand & & $\begin{array}{c}2.288^{* * *} \\
(0.824) \\
\end{array}$ & $\begin{array}{c}2.453^{* * *} \\
(0.837) \\
\end{array}$ \\
\hline Domain Effects & No & No & Yes \\
\hline Demographics & No & No & Yes \\
\hline Log Likelihood & $-17,982.730$ & -838.230 & -685.891 \\
\hline
\end{tabular}

well), ads should mainly focus on brand image building while for non-experienced users, ads should be informative.

\section{DISCUSSION}

To our knowledge, this is the first empirical study that combines sales data and search data to examine post-purchase searches. The results of this paper are unsurprising ex-post: (1) for many core branded keywords, more than half of searchers own the product, and (2) post-purchase searchers are responsive to advertisements, but (3) they should be shown advertisements tailored to post-purchase needs such as those for complementary products. However, the unique data required this study was unfeasible until now, and the actions of key stakeholders such as advertisers and search engine designers imply that the results were not obvious prior to our research. The volume and intents of searches in the days after purchase are not obvious to advertisers, whose advertisements are tailored to prospective consumers. The newly available functionality to target or exclude tracked, post-purchase searchers is largely marketed as a way to exclude current owners from targeted campaigns. However, as the group of current users is both very large and susceptible to the right advertisements, this suggests that advertising platforms are unaware of the number of post-purchase searchers and the possibilities to reach them. We expect our findings to inspire more researchers, both in academic and industry settings, to focus on improving advertisement efficiency, maximize revenue for search engines, and increase search engine users' welfare.

Our research extends to several other product spaces (withheld from this study due to space constraints), including online media (Hulu, Netflix), online services (Ancestry, Natch), and automobiles (Ford, Toyota). The results from these areas largely validate the present study. We consistently see high percentages of postpurchase searches made for branded keywords. We also always see significant differences in what users click on, conditional on being post purchase. However, at this time, the randomization in advertisements required to conduct studies that are as in-depth as the one we present here is not available for all products. It is possible that for some products and services, post-purchase searches have higher ads click through rate because current users are likely to use the ads link for navigation purposes, which supports the findings in /citeBlake2015. Here we acknowledge that pre and post-purchase searches have different needs and behaviour, but the pattern of the difference is contingent on the type of products, services or even advertisement message that we show to them.

If anything, our results underestimate the quantity of post-purchase searchers, which creates a downward bias regarding the impact of our results. Errors in identifying post-purchase searchers are almost entirely false negatives. While it is rare for our procedures to identify a searcher who owns a product when they do not own it in actuality, it is common to miss off-line purchases or purchases made in an unidentified online setting. Thus, many searches identified as pre-purchase are actually post-purchase, somewhat mooting the differences between the two sets of searchers. This will likely be the case for any practical use of advertising platforms moving forward, so we accept this as a limitation of both the study and its real-world analog.

This is frightening research for search engines, as it could result in the recommendation to not advertise to searchers post-purchase, which would be devastating to revenue. Our study suggests that that is not the case, but we feel that it is critical to explore the work further because post-purchase search comprises a huge portion of a large industry. We look forward to extending the research into advertising revenue to better understand the implications for 
advertising platforms of separating out pre- and post-purchase searches.

This is exciting research for advertisers seeking to formulate an efficient strategy, as it is possible to identify post-purchase searchers within a query. There has been insufficient academic research on how advertisers should use these functions or whether they should use them at all. Our results can help advertisers strategically plan their advertisement budget. We look forward to extending the research into customer conversation and return costs to better capture the trade-offs of adverting to post-purchase searchers.

This is also meaningful research for searchers who may be frustrated by the post-purchase search experience. Search advertising is geared towards pre-purchase, with an emphasis on the initial purchase of units. Post-purchase needs are more varied, ranging from complementary items to product help. This makes both advertising and organic linkage difficult. We hope to extend our research to gain a better understanding of the social welfare implications for the users of these platforms by better identifying them and accordingly discriminating content targeted to them.

This work will become increasingly critical to platforms, advertisers, and consumers as returns become easier and more purchases are not only made online but also consumed online. It is estimated that product returns in the US have reached over $\$ 100$ billion per year [9], with annual growth as high as $21 \%$ according to Accenture. Although product returns are considered in some retail business models, this still imposes a huge financial burden on manufactures and retailers and negatively influences brand image. Thus, understanding consumers' needs and concerns after purchase can also help retailers to reduce product returns.

There are a number of limitations to the current study. By using found randomization of advertising, the distribution of links is less than optimal; we look forward to future studies in which we can make different choices to optimize results. Further, we are constantly updating how we identify purchase and look forward to reducing the asymmetric identification of searchers in the purchase funnel.

\section{REFERENCES}

[1] Daniel A Ackerberg. 2001. Empirically distinguishing informative and prestige effects of advertising. RAND fournal of Economics (2001), 316-333.

[2] Animesh Animesh, Vandana Ramachandran, and Siva Viswanathan. 2010. Research note - Quality uncertainty and the performance of online sponsored search markets: An empirical investigation. Information Systems Research 21, 1 (2010), 190-201.

[3] Thomas Blake, Chris Nosko, and Steven Tadelis. 2015. Consumer heterogeneity and paid search effectiveness: A large-scale field experiment. Econometrica 83, 1 (2015), 155-174.

[4] Andrei Broder. 2002. A taxonomy of web search. In ACM Sigir forum, Vol. 36 ACM, 3-10.

[5] Tat Y. Chan, Chunhua Wu, and Ying Xie. 2011. Measuring the Lifetime Value of Customers Acquired from Google Search Advertising. Marketing Science 30, 5 (2011), 837-850. https://doi.org/10.1287/mksc.1110.0658

[6] Henry A Feild, James Allan, and Rosie Jones. 2010. Predicting searcher frustration. In Proceedings of the 33rd international ACM SIGIR conference on Research and development in information retrieval. ACM, 34-41.

[7] Anindya Ghose and Sha Yang. 2009. An Empirical Analysis of Search Engine Advertising: Sponsored Search in Electronic Markets. Management Science 55, 10 (2009), 1605-1622. https://doi.org/10.1287/mnsc.1090.1054

[8] Cristina González-Caro and Ricardo Baeza-Yates. 2011. A multi-faceted approach to query intent classification. Lecture Notes in Computer Science (including subseries Lecture Notes in Artificial Intelligence and Lecture Notes in Bioinformatics) 7024 LNCS (2011), 368-379. https://doi.org/10.1007/978-3-642-24583-1_36
[9] V. Daniel R. Guide, Gilvan C. Souza, Luk N. Van Wassenhove, and Joseph D. Blackburn. 2006. Time Value of Commercial Product Returns. Management Science 52, 8 (2006), 1200-1214. https://doi.org/10.1287/mnsc. 1060.0522

[10] Oi Guo and Eugene Agichtein. 2010. Ready to buy or just browsing?: detecting web searcher goals from interaction data. In Proceedings of the 33rd international ACM SIGIR conference on Research and development in information retrieval. ACM, $130-137$.

[11] Qi Guo and Eugene Agichtein. 2012. Beyond dwell time: estimating document relevance from cursor movements and other post-click searcher behavior. In Proceedings of the 21st international conference on World Wide Web. ACM, 569-578.

[12] Ahmed Hassan, Rosie Jones, and Kristina Lisa Klinkner. 2010. Beyond DCG: user behavior as a predictor of a successful search. In Proceedings of the third ACM international conference on Web search and data mining. ACM, 221-230.

[13] Ahmed Hassan and Ryen W. White. 2013. Personalized models of search satisfaction. Proceedings of the 22nd ACM international conference on Conference on information \\& knowledge management - CIKM '13 (2013), 2009-2018. https://doi.org/10.1145/2505515.2505681

[14] I Hernández, Parth Gupta, Paolo Rosso, and Martha Rocha. 2012. A simple model for classifying web queries by user intent. In Proc. 2nd Spanish Conf. Information Retrieval. 235-240.

[15] PwC IAB. 2016. IAB Internet advertising revenue report 2016 full-year results. Technical Report. Market research report, Interactive Advertising Bureau (IAB) and PricewaterhouseCoopers $(\mathrm{PwC})$

[16] Bernard J Jansen, Danielle L Booth, and Amanda Spink. 2007. Determining the user intent of web search engine queries. In Proceedings of the 16th international conference on World Wide Web. ACM, 1149-1150.

[17] Bernard J Jansen and Simone Schuster. 2011. Bidding on the Buying Funnel for Sponsored Search and Keyword Advertising. Journal of Electronic Commerce Research 12, 1 (2011), 1-18.

[18] Kinshuk Jerath, Liye Ma, and Young-Hoon Park. 2014. Consumer click behavior at a search engine: The role of keyword popularity. fournal of Marketing Research 51, 4 (2014), 480-486.

[19] Ashish Kathuria, Bernard J. Jansen, Carolyn Hafernik, and Amanda Spink. 2010. Classifying the user intent of web queries using k-means clustering. Internet Research 20, 5 (2010), 563-581. https://doi.org/10.1108/10662241011084112

[20] David Kempe and Brendan Lucier. 2014. User satisfaction in competitive sponsored search. In Proceedings of the 23rd international conference on World wide web. ACM, 699-710.

[21] Youngho Kim, Ahmed Hassan, Ryen W White, and Imed Zitouni. 2014. Modeling dwell time to predict click-level satisfaction. In Proceedings of the 7th ACM international conference on Web search and data mining. ACM, 193-202.

[22] Uichin Lee, Zhenyu Liu, and Junghoo Cho. 2005. Automatic identification of user goals in web search. In Proceedings of the 14th international conference on World Wide Web. ACM, 391-400.

[23] Hongshuang (Alice) Li, P. K. Kannan, Siva Viswanathan, and Abhishek Pani. 2016. Attribution Strategies and Return on Keyword Investment in Paid Search Advertising. Marketing Science 35, 6 (nov 2016), 831-848. https://doi.org/10. $1287 / \mathrm{mksc} .2016 .0987$

[24] Wendy W. Moe. 2003. Buying, Searching, or Browsing: Differentiating Between Online Shoppers Using In-Store Navigational Clickstream. Fournal of Consumer Psychology 13, 1-2 (2003), 29-39. https://doi.org/10.1207/S15327663JCP13-1\&2_ 03

[25] Alaa Mohasseb, Maged El-Sayed, and Khaled Mahar. 2014. Automated Identification of Web Queries using Search Type Patterns. In WEBIST (2). 295-304.

[26] Oliver J Rutz and Randolph E Bucklin. 2011. From generic to branded: A model of spillover in paid search advertising. Journal of Marketing Research 48, 1 (2011), 87-102.

[27] Oliver J Rutz, Michael Trusov, and Randolph E Bucklin. 2011. Modeling indirect effects of paid search advertising: Which keywords lead to more future visits? Marketing Science 30, 4 (2011), 646-665.

[28] Andrey Simonov, Chris Nosko, and Justin M Rao. 2018. Competition and crowdout for brand keywords in sponsored search. Marketing Science (2018).

[29] Thomas. Stern. [n. d.]. Trend Observation: Branded PPC Ads Getting More Expensive. ([n. d.]). searchengineland.com/ trend-observation-branded-ppc-ads-experience-increased-costs- 247832 .

[30] Ryen W White and Susan T Dumais. 2009. Characterizing and predicting search engine switching behavior. In Proceedings of the 18th ACM conference on Information and knowledge management. ACM, 87-96.

[31] S. Yang and A. Ghose. 2011. Analyzing the Relationship Between Organic and Sponsored Search Advertising: Positive, Negative, or Zero Interdependence? Marketing Science 30, 1 (jan 2011), v-v. https://doi.org/10.1287/mksc.1100.0552 